\title{
Application of Model predictive control suitable for closed loop re-identification to a polymerization reactor
}

\author{
Germán A. Bustos $^{\dagger}$, Alejandro H. González ${ }^{\dagger}$, Antonio Ferramosca ${ }^{\dagger}$ and Jacinto L. Marchetti ${ }^{\dagger}$ \\ $\dagger$ Institute of Technological Development for the Chemical Industry (INTEC) \\ CONICET Universidad Nacional del Litoral (UNL). \\ Güemes 3450, (3000) Santa Fe, Argentina. gbustos, alejgon, ferramosca, jlmarch@santafe-conicet.gov.ar
}

\begin{abstract}
Recently, a Model Predictive Control (MPC) suitable for closed-loop re-identification was proposed ([1]). This strategy try to solve the potential conflict between the persistent excitation of the system - necessary to perform a suitable identification and the stabilizing control objectives. The main idea is to extend the concept of equilibrium-point-stability to the invariant-set-stability, and propose an MPC that: steers the system to that invariant set, when outside, and persistently excite the system, when inside. In this work, an application of this MPC to a nonlinear polymerization reactor simulator is made to explore the difficulties arising from the intention to approach real life problems. Several scenarios are simulated in order to clearly show the benefits and limitations of the proposed MPC strategy.
\end{abstract}

Keywords-Model predictive control, closedloop identification, polymerization reactor, persistent excitation.

\section{Introduction}

Model predictive control (MPC) is typically implemented as a lower stage of a hierarchical control structure. The upper level stages are devoted to compute, by means of a stationary optimization, the targets that the dynamic control stage (MPC) should reach to economically optimize the operation of the process. Since both, the dynamic and stationary optimizations are model based optimizations, a periodic updating of the model parameters are desired to reach meaningful optimums. In this context, a re-identification procedure should be developed in a closed-loop fashion, since the process cannot be stopped each time an update is needed. As it is known, the main problem of a closed-loop identification is that the dynamic control objectives are incompatible with the identification objectives. In fact, to perform a suitable identification, a persistent excitation of the system modes is needed, while the controller takes this excitation as disturbance that it tries to reject from the output to stabilize the system.

The closed-loop identification methods fall into the following main groups ([2]). The direct approach ignores the feedback law and identifies the open-loop system using measurements of the input and the output. The in- direct approach identifies the closed-loop transfer function and determines the open-loop parameters subtracting the controller dynamic. To do that, controller dynamic must be linear and known. The joint input-output approach takes the input and output jointly, as the output of a system produced by some extra input or set-point signal. Since the last two methods needs the exact knowledge of a linear controller, they are not directly applicable for closed-loops under constrained MPC controllers.

Several strategies were developed to perform closedloop re-identification under MPC controllers: [3] proposed a controller named Model Predictive Control and Identification (MPCI) where a persistent excitation condition is added by means of an additional constraints in the optimization problem. This strategy, which was explored later in ([4]), turns the MPC optimization problem non-convex, and so, most of the well-known properties of the MPC formulation cannot be established. [5] proposed a strategy that manipulates the steady state target optimization (in the hierarchical MPC control structure) in order to excite the system. This strategy does not modify the MPC optimization problem structure, but the identification is performed on the whole closed-loop system. [6] proposed a simpler closed-loop identification strategy to estimate multivariable process gains directly from on-line data. The control structure adopted to test the proposed strategy is an LP-MPC control structure frequently used in industrial applications. In general, none of these reports have shown results regarding the stability of the MPC while it is re-identifying the system. In this work, based on the concept of stability of an invariant set (as a generalization of an equilibrium point), a MPC controller with a extended domain of attraction is proposed, which assures both, stability and persistent excitation conditions, in order to perform a safe closed-loop re-identification.

Recently, [1] have proposed a novel MPC suitable for re-identification. The main idea is to extend the concept of equilibrium-point-stability to the invariant-setstability, and proposed an MPC that: steers the system to that invariant set, when outside, and persistently excite the system, when inside. The MPC problem formulation is based on the concept of generalized distance from a point (the state and input trajectory) to a set (target invari- 
ant set and input excitation set). So, it guaranties stability of the target invariant set and also the persistent excitation of the system, since both task are developed separately.

The idea of this work is to show the application and to evaluate the practical benefits of the MPC suitable for re-identification [1] in a styrene polymerization reactor. This kind of application in a real complex system is not trivial and many difficulties, not accounted in [1], arise, as can be seen next.

Notation: Matrices $I_{n} \in \mathbb{R}^{n \times n}$ and $0_{n, m} \in \mathbb{R}^{n \times m}$ denote the identity matrix and the null matrix, respectively. A $C$-set is a convex and compact set that contains the origin. A proper $C$-set is a C-set that contains the origin as an interior point. Consider two sets $\mathcal{U} \subseteq \mathbb{R}^{n}$ and $\mathcal{V} \subseteq \mathbb{R}^{n}$, containing the origin, and a real number $\lambda$. The Minkowski sum $\mathcal{U} \oplus \mathcal{V} \subseteq \mathbb{R}^{n}$ is defined by $\mathcal{U} \oplus \mathcal{V}=\{(u+v): u \in \mathcal{U}, v \in \mathcal{V}\}$; the set $(\mathcal{U} \backslash \mathcal{V}) \subseteq \mathbb{R}^{n}$ is defined by $\mathcal{U} \backslash \mathcal{V}=\{u: u \in \mathcal{U} \wedge u \notin \mathcal{V}\}$; and the set $\lambda \mathcal{U}=\{\lambda u: u \in \mathcal{U}\}$ is a scaled set of $\mathcal{U}$.

\section{Problem statement and preliminaries}

Consider a system described by a linear time-invariant discrete-time model

$$
\begin{aligned}
x^{+} & =A x+B u \\
y & =C x
\end{aligned}
$$

where $x \in \mathbb{R}^{n}$ is the system state, $x^{+}$is the successor state, $u \in \mathbb{R}^{m}$ is the current control, and $y \in \mathbb{R}^{p}$ is the system output. The state, the control input and the output at discrete-time instant $k$ are denoted as $x(k), u(k)$ and $y(k)$, respectively. The solution of model (1), for a given sequence of control inputs $\mathbf{u}=\{u(0), \cdots, u(j-1)\}$ and an initial state $x$ is denoted as: $x(j)=\phi(j ; x, \mathbf{u})=$ $A^{j} x(0)+\sum_{i=0}^{j-1} A^{j-i+1} B u(i), j \in \mathbb{I}_{\geq 1}$.

The system is subject to constraints on state and input:

$$
(x(k), u(k)) \in \mathcal{Z} \triangleq(\mathcal{X} \times \mathcal{U}) \subset \mathbb{R}^{n+m}
$$

for all $k \geq 0$, where $\mathcal{X} \subset \mathbb{R}^{n}$ and $\mathcal{U} \subset \mathbb{R}^{m}$. It is assumed that:

Assumption 1. Matrix $A$ has all its eigenvalues strictly inside the unit circle, the pair $(A, B)$ is controllable and the state is measured at each discrete-time instant. Furthermore, the set $\mathcal{X}$ is convex and closed, the set $\mathcal{U}$ is convex and compact and both contain the origin in their interior.with $\lambda \in[0,1)$.

The main objective of this work is to show the application of an MPC controller suitable for closed-loop reidentification in a styrene polymerization reactor, ensuring at the same time a generalized stability. The proposed controller has two modes; when no identification is needed, the controller will maintain the closed-loop stability of a desired equilibrium set (zone control). When a reidentification of the model is needed, the controller maintain the closed-loop stability of an (appropriate defined) invariant set, and once the system is inside the invariant set, a persistent excitation signal is applied.

\section{The MPC suitable for closed-loop re-identification}

In this section we briefly described the MPC formulation presented in [1]. As was already said, the main idea of that work is to extend the concept of equilibrium-pointstability to the invariant-set-stability, and to propose an MPC that: steers the system to that invariant set, when outside, and persistently excite the system, when inside. In this context, and before proceed with the MPC formulation, we describe the main sets involved in the formulation.

\subsection{Description of the main sets involved in the formulation}

As it is known, in order to estimate a model from measured input and output data, each (controllable) mode of the system must be excited. To do that, the excitation input signal should contain enough variability. This property is generally indicated by the notion of persistency of excitation ([7]). So, we can define now a persistent excitation (PE) sequence, $\mathbf{u}_{P E}=\left\{u_{P E}(0), \cdots, u_{P E}\left(T_{i d}\right)\right\}$, $u_{P E}(j) \in \mathbb{R}^{m}$, for $j \in \mathbb{I}_{0: T_{i d}}$, where $T_{i d}$ is the length of the data necessary to perform a suitable identification.

The PE input might be of several forms, going from a Pseudo-Random Binary Signal (PRBS) signal to a Filtered Pseudo Gaussian White Noise Signal, but should be bounded (since unbounded input signal has not physical meaning). Then, we can define a compact excitation input set where the PE sequence will be contained.

Definition 1. (Excitation input set, EIS). An input proper $C$-set $\mathcal{U}^{t} \subset \mathcal{U}$, which contains the persistent excitation sequence PE, i.e., $u_{P E}(j) \in \mathcal{U}^{t}$, for $j \in \mathbb{I}_{0: T_{i d}}$, will be denoted as excitation input set.

The EIS set $\mathcal{U}^{t}$ defines a set $\mathcal{Z}^{t} \triangleq\left(\mathcal{X} \times \mathcal{U}^{t}\right) \subset \mathbb{R}^{n+m}$ in the state-input space. Notice that the EIS set (volume) comes from reality, since in real plants the noise in the variables determines a minimal amplitude for the input excitation. Now, the bounded nature of the EIS set $\mathcal{U}^{t}$ and the random nature of the persistent excitation input defines an autonomous system given by:

$$
x^{+}=A x+B u_{P E} \triangleq f_{P E}(x),
$$

where $u_{P E} \in \mathcal{U}^{t}$ are the inputs of the persistent excitation sequence $\mathbf{u}_{P E}$. With these concepts in mind we can now define:

Definition 2. ( $\lambda$-Invariant set for identification, $\lambda$-ISI) A proper $C$-set $\mathcal{X}^{t} \subseteq \mathcal{X}$ is a $\lambda$-invariant set for identification, with $\lambda \in[0,1]$, for the non autonomous system $x^{+}=A x+B u,(x, u) \in \mathcal{Z}^{t}$, if $x \in \mathcal{X}^{t}$ implies $x^{+} \in \lambda \mathcal{X}^{t}$, for all $u \in \mathcal{U}^{t}$.

A $\lambda$-ISI set with $\lambda=1$ is simply an ISI set. Clearly, an ISI set definition is a robust invariant set definition adapted to identification requirements. So, we can define the smallest ISI set, for a given system, which is given by the minimal ISI set ([8]): $\underline{\mathcal{X}}^{t}=\bigoplus_{i=0}^{\infty}\left(A^{i} B \mathcal{U}^{t}\right)$. 


\subsection{MPC problem formulations}

The MPC problem formulation is based on the concept of generalized distance from a point (the state and input trajectory) to a set (target invariant set and input excitation set). So, it guaranties stability of the target invariant set and also the persistent excitation of the system, since both task are developed separately.

The complete MPC strategy proposed in [1] has two modes: one that operates when no re-identification is nedeed, and is defined as MPC for tracking control equilibrium sets, and other, that operates when reidentification is nedeed, which is defined as MPC suitable for re-identification.

\subsubsection{First mode}

The first of this controllers cost is formulated following a similar strategy to the proposed in $[9,10]$, usually known as zone control:

$$
\begin{aligned}
V_{N}^{C E S}\left(x, \mathcal{X}_{s s}^{t} ; \mathbf{u}\right) & =\sum_{j=0}^{N-1}\left(\left\|x(j), x_{s s}\right\|_{Q}^{2}\right. \\
& \left.+\left\|u(j), u_{s s}\right\|_{R}^{2}\right)+\gamma d_{\mathcal{X}_{s s}^{t}}(x(s s)),
\end{aligned}
$$

where $Q>0$ and $R \geq 0$ are penalization matrices, $\gamma>0$ is a real number, $d_{\mathcal{X}_{s s}^{t}}(x(s s))$ is the generalized distance function (from $x_{s s}$ to $\mathcal{X}_{s s}^{t}$ ), $\mathcal{X}_{s s}^{t}$ is a control equilibrium set and $N$ is the control horizon. Furthermore, the state $x_{s s} \in \mathcal{X} s s=G B \mathcal{U}$ represents the stationary value corresponding to $u_{s s}$. For any current state $x \in \mathcal{X}$, the optimization problem $P_{N}^{C E S}\left(x, \mathcal{X}_{s s}^{t}\right)$ to be solved is given by:

$$
\begin{aligned}
\text { Problem } P_{N}^{C E S}\left(x, \mathcal{X}_{s s}^{t}\right) & \\
\operatorname{mín}_{\mathbf{u}} & V_{N}^{C E S}\left(x, \mathcal{X}_{s s}^{t} ; \mathbf{u}\right) \\
\text { s.t. } & \\
& x(0)=x, \\
& x(j+1)=A x(j)+B u(j), \quad j \in \mathbb{I}_{0: N-1} \\
& x(j) \in \mathcal{X}, u(j) \in \mathcal{U}, \quad j \in \mathbb{I}_{0: N-1} \\
& u(j)=u_{s s}, \\
& x_{s s}=G B u_{s s} .
\end{aligned}
$$

In this optimization problem, $x$ and $\mathcal{X}_{s s}^{t}$ are the parameters, while the sequence $\mathbf{u}=\{u(0), \cdots, u(N-1)\}$ and $x_{s s}, u_{s s}$, are the optimization variables. The control law, derived from the application of a receding horizon policy, is given by $\kappa_{N}\left(x, \mathcal{X}_{s s}^{t}\right)=u^{0}(0 ; x)$, where $u^{0}(0 ; x)$ is the first element of the solution sequence $\mathbf{u}^{0}(x)$.

Now it can be established the following Theorem:

Theorem 1. Consider that assumptions 1 hold, and consider a given a control equilibrium set $\mathcal{X}_{s s}^{t}=G B \mathcal{U}^{t} \subseteq$ $\mathcal{X}$, with an associated input set $\mathcal{U}^{t}$. Then, $\mathcal{X}_{s s}^{t}$ is an equilibrium set for the closed-loop system $x^{+}=A x+$ $B \kappa_{N}\left(x, \mathcal{X}_{s s}^{t}\right)$. Furthermore, $\mathcal{X}_{s s}^{t}$ is locally attractive for the closed-loop system $x^{+}=A x+B \kappa_{N}\left(x, \mathcal{X}_{s s}^{t}\right)$, with a domain of attraction given by $\mathcal{X}$.

Demostración. The proof can be seen in $[9,10]$.

\subsubsection{Second mode}

The second controller (MPC suitable for reidentification) has a cost given by:

$$
\begin{aligned}
V_{N}^{E X C}\left(x, \mathcal{X}^{t}, \mathbf{u}_{P E}, k ; \mathbf{u}\right) & =(1-\rho(x)) V_{N}^{I S I}\left(x, \mathcal{X}^{t} ; \mathbf{u}\right) \\
& +\rho(x)\left\|u(0)-u_{P E}(k)\right\|,
\end{aligned}
$$

where $\rho(x)=1$ if $x \in \mathcal{X}^{t}$, and $\rho(x)=0$, otherwise. Furthermore, $V_{N}^{I S I}\left(x, \mathcal{X}^{t} ; \mathbf{u}\right)$ is given by:

$$
\begin{aligned}
V_{N}^{I S I}\left(x, \mathcal{X}^{t} ; \mathbf{u}\right) & =\sum_{j=0}^{N-1}\left(\alpha d_{\mathcal{X}^{t}}(x(j))+\beta d_{\mathcal{U}^{t}}(u(j))\right) \\
& +d_{\mathcal{X}^{t}}^{t e r}(x(N)) .
\end{aligned}
$$

where $\alpha$ and $\beta$ are positive real numbers, $d_{\mathcal{X}}(x)$ is a generalized distance from point $x$ to set $\mathcal{X}$ and $d_{\mathcal{X}^{t}}^{t e r}(\cdot)$ is a terminal cost function defined on $\mathcal{X}^{\text {ter }}$, which is the terminal set where the terminal state $x(N)$ is forced to belong.

For any initial state $x$ in the $N$-step controllable set to $\mathcal{X}^{\text {ter }}\left(\mathcal{X}_{N}\left(\mathcal{X}^{\text {ter }}\right)\right)$, at a given time step $k$, the optimization problem $P_{N}^{E X C}\left(x, \mathcal{X}^{t}, \mathbf{u}_{P E}, k\right)$, to be solved at each time instant $k$, is given by:

$$
\begin{array}{cl}
\text { Problem } P_{N}^{E X C}\left(x, \mathcal{X}^{t}, \mathbf{u}_{P E}, k\right) \\
\min _{\mathbf{u}} & V_{N}^{E X C}\left(x, \mathcal{X}^{t}, \mathbf{u}_{P E}, k ; \mathbf{u}\right) \\
\text { s.t. } & \\
& x(0)=x, \\
& x(j+1)=A x(j)+B u(j), \quad j \in \mathbb{I}_{0: N-1} \\
& x(j) \in \mathcal{X}, u(j) \in \mathcal{U}, \quad j \in \mathbb{I}_{0: N-1} \\
& x(N) \in \mathcal{X}^{\text {ter }}
\end{array}
$$

The controller derived from this formulation assures the convergence of the closed-loop system to the $\lambda$-ISI set $\mathcal{X}^{t}$, and once the system is there, the persistent excitation of the system is performed. The following Theorem formalizes the properties of the proposed MPC controller:

Theorem 2. Consider that assumption 1 holds, and consider an ISI set $\mathcal{X}^{t}$ and a persistent excitation sequence $\mathbf{u}_{P E}$ contained in the EIS set $\mathcal{U}^{t}$. Then, for any initial state $x \in \mathcal{X}^{t}$, the system controlled by the receding horizon MPC control law $\kappa_{N}\left(x, \mathcal{X}^{t}\right)=u^{0}(0 ; x)$, will be persistently excited inside $\mathcal{X}^{t}$ (i.e, $x^{+}=A x+$ $\left.B \kappa_{N}\left(x, \mathcal{X}^{t}\right)=f_{P E}(x)\right)$. Furthermore, for any initial state $x \in \mathcal{X}_{N}\left(\mathcal{X}^{\text {ter }}\right) \backslash \mathcal{X}^{t}$, the closed-loop converges to $\mathcal{X}^{t}$.

Demostración. The proof can be seen in [1]

\subsection{Operation of the loop}

Based on the above discussion, the MPC controller operation will be presented.

(1) Control operation mode: in this mode no reidentification is needed, and the MPC for tracking control equilibrium sets is implemented (Problem $\left.P_{N}^{C E S}\left(x, \mathcal{X}_{s s}^{t}\right)\right)$, and 
(2) Re-identification operation mode: this mode is activated only when there is a suspect that the model is not working properly, and a re-identification is needed. In this mode the persistent excitation MPC is used (Problem $\left.P_{N}^{E X C}\left(x, \mathcal{X}^{t}, \mathbf{u}_{P E}, k\right)\right)$.

\subsection{Model mismatch}

It should be noticed that the ISI target set $\mathcal{X}^{t}$, which is a parameter of the MPC optimization cost, depends on the model. Since the excitation scenario is precisely given when we suspect that the current model is no longer accurate, a discussion about the effect of the model mismatch on the computation of $\mathcal{X}^{t}$ is needed. Next, a robust ISI sets $\mathcal{X}^{t}$ designs will be presented.

\subsubsection{Parametric uncertainty}

Consider a system given by

$$
x^{+}=A(w) x+B(w) u, \quad y=C(w) x,
$$

where $A(w)$ and $B(w)$ are affine functions of $w$, i.e., $A(w)=A+\bar{A} w, B(w)=B+\bar{B} w$ and $w$ belongs to the proper $\mathrm{C}$-set $\mathcal{W} \subset \mathbb{R}$. Furthermore, assume that the Nominal model is given by $x^{+}=A\left(w_{N}=0\right) x+B\left(w_{N}=\right.$ $0) u=A x+B u$, and the unknown Real model, is given by $x^{+}=A\left(w_{R}\right) x+B\left(w_{R}\right) u$, for some $w_{R} \in \mathcal{W}$. In this context, there is a minimal ISI set $\underline{\mathcal{X}}^{t}(w)$ associated to each particular model. Now, consider the following Theorem:

Theorem 3. Consider a $\lambda$-ISI set $\mathcal{X}^{t}(w=0), \lambda \in[0,1)$, for $x^{+}=A x+B u,(x, u) \in \mathcal{Z}^{t}$. Then, there exists a non-empty set $\mathcal{W} \subset \mathbb{R}$ for which the set $\mathcal{X}^{t}(w=0)$ is an ISI set for $x^{+}=A(w) x+B(w) u,(x, u) \in \mathcal{Z}^{t}$, for all $w \in \mathcal{W}$.

Demostración. The proof can be seen in [1].

\section{The styrene polymerization reactor}

The polymerization reactor is usually the heart of the polymer production process and its operation may be difficult as it involves exothermic reactions, unknown reaction kinetics and high viscosity [11]. Most styrene polymers are produced through batch or continuous polymerization processes. The present work considers the free-radical bulk and solution styrene polymerization in a jacketed CSTR. The CSTR has three feed streams: the pure styrene monomer, the 2,2'-azoisobutyronitrile (AIBN) initiator dissolved in benzene, and the pure benzene solvent. The exit stream contains polymer, unreacted monomer, initiator, and solvent. The kinetic mechanism used for this homopolymerization process is quite general and is described in [12]: Furthermore, [13] and [14] developed a phenomenological model - based on a list of consideration that can be seen in these works - for the styrene reactor, that was the one used in [11]. The phenomenological model of this styrene reactor was first published in 1990 and, since then, it has been widely used as a benchmark for process control studies In [11], the aim is to optimize on-line the production rate using a hierarchical structure. The real time optimization is developed in the upper stage and an intermediary routine recalculates the optimizing targets which are sent to the MPC controller. These stages are tied together and one needs to guarantee the stability of the complete control structure. The definition and values of the parameters and variables involved in the phenomenological model can be found in [11], that was the work we based on to apply the MPC controller.

\subsection{Linear Models for predictions}

Here, the average molecular weight $\bar{M}_{W}$ and the reactor temperature $\mathrm{T}$ are defined as the controlled outputs. As on-line measurements of $\bar{M}_{W}$ are rarely available, the polymer intrinsic viscosity $\eta$ is used instead. For controlling $y_{1}=\eta$ and $y_{2}=T$, the controller manipulates the initiator flowrate $\left(u_{1}=Q_{i}\right)$ and the liquid flow rate of the cooling jacket $\left(u_{2}=Q_{c}\right)$ because of the adequate sensitivity of the process outputs to these variables. The remaining inlet flowrates $Q_{s}$ and $Q_{m}$ are related to $Q_{i}$ by ratio control. So as to improve the performance of the controller, the ratio between the initiator flow rate $Q_{i}$ and monomer flow rate $Q_{m}$ is maintained fixed, then:

$$
Q_{m}=\frac{\bar{Q}_{m}}{\bar{Q}_{i}} Q_{i}
$$

where $\bar{Q}_{m}$ and $\bar{Q}_{I}$ are the nominal values of $Q_{m}$ and $Q_{i}$, respectively. On the other hand, the solvent volume fraction should be maintained at 0.6 to avoid the gel effect [13], then a control law for the solvent flow rate is implemented as:

$$
Q_{s}=1,5 Q_{m}-Q_{i}
$$

The control structure considered for the styrene reactor requires linear models. The nominal model used for prediction is the following state space model:

$$
\begin{aligned}
& A=\left[\begin{array}{ccc}
0,9090 & -0,1206 & -0,0378 \\
-0,1196 & 0,5992 & -0,1527 \\
0,0397 & -0,0392 & 0,8373
\end{array}\right], \\
& B=\left[\begin{array}{cc}
-3,9153 & 0,9745 \\
2,0845 & -1,3483 \\
1,0877 & 1,0609
\end{array}\right], \\
& C=\left[\begin{array}{ccc}
1,5359 & 2,3478 & -0,5689 \\
-3,6469 & -2,6701 & -1,6449
\end{array}\right], \\
& D=\left[\begin{array}{cc}
0,0499 & 3,0581 \\
-1,0722 & 1,1369
\end{array}\right],
\end{aligned}
$$

This model relates process inputs and outputs that was obtained at the nominal operating point $u_{s s}^{1}$ and $y_{s s}^{1}$ presented in the next section.

\section{Simulation results}

In this section some simulations results will be presented, to evaluate the proposed control strategy in the described polymerization reactor. The operating points are 


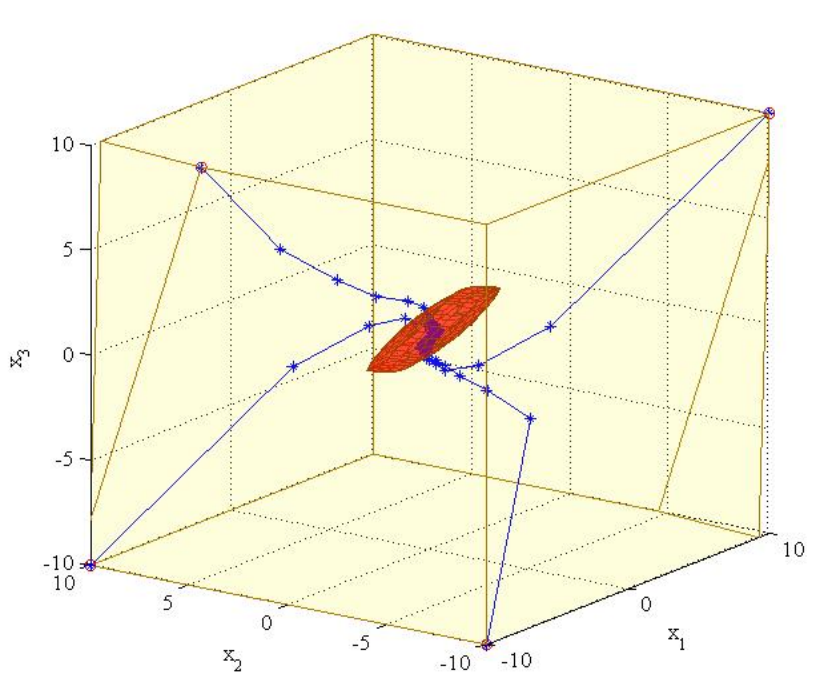

Figura 1: State evolution corresponding to several initial states.

assumed to be known (they come form an upper-level RTO optimization problem) and two scenarios will be simulated.

\subsection{Nominal case}

First, we assume an operating point given by $u_{s s}^{1}=$ $[0,0300,131]$ and $y_{s s}^{1}=[3,8968323,56]$. For this operating point, (7) to (10) is used as a nominal model of the MPC. The state and input constraints (that was centered at the operating point), $\mathcal{X}$ and $\mathcal{U}$, are given by $[-10-10-10]^{T} \leq x \leq\left[\begin{array}{lll}10 & 10 & 10\end{array}\right]^{T}$, $[-0,015-0,051]^{T} \leq u \leq[0,040,119]^{T}$, respectively. The excitation input set (EIS), $\mathcal{U}^{t}$, has been selected to be $[-0,0060-0,0204]^{T} \leq u \leq[0,01600,0476]^{T}$, while the persistent excitation signal was selected to be a Pseudo Random Binary Signal (PRBS). The $\lambda$-ISI set, $\mathcal{X}^{t}$, was computed according to $\mathcal{U}^{t}$. Figure 1 shows the relation between the feasible state space $\mathcal{X}$ (in light yellow) and the invariant set for identification (ISI) $\mathcal{X}^{t}$ (in red).

The performed simulations were designed to show the Re-identification operation mode of the controller. As can be seen in Figure 1, every (feasible) state in $\mathcal{X} \backslash \mathcal{X}^{t}$ is steered to the target set $\mathcal{X}^{t}$, and once the system is inside this set, the exciting procedure is activated.

Figure 2, 3 and 4 shows the input, outputs and cost function time evolutions respectively. Notice that there are two clear modes: first, from time $k=0$ to time $k=6$, the system is steered to the ISI set, with a decreasing cost function. Then, from time $k=6$ on, the cost function remains null, which corresponds to a persistent excitation determined by the signal $\mathbf{u}_{P E}$. Notice also, that the inputs are on the bounds in the first time periods, because the controller tries to do the best for the system to reach the target. Furthermore, after time $k=6$, the input remain
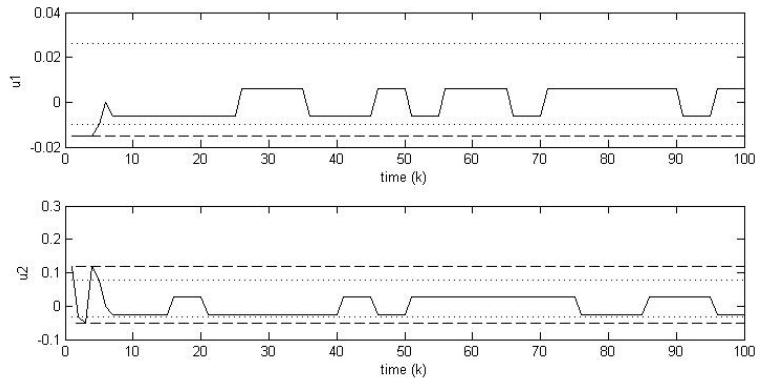

Figura 2: Input evolution.
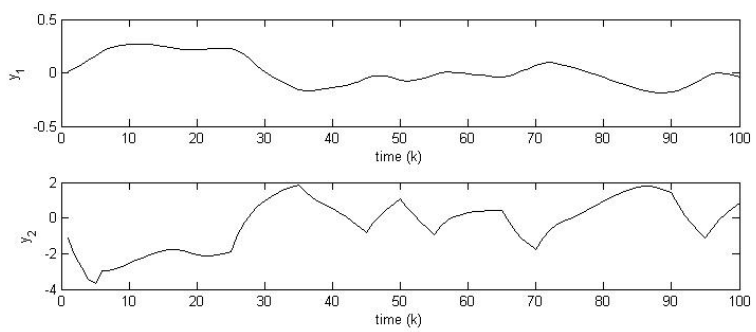

Figura 3: Output evolution.

inside the EIS set $\mathcal{U}^{t}$, denoted by two dotted-lines.

\subsection{Model-mismatch case}

In this section a simulation result is presented to evaluate the proposed control strategy in a model uncertainty scenario. To this end, the operation point of the system was change - based on the work of [11] - to $u_{s s}^{2}=$ $[0,03300,1245]$ and $y_{s s}^{2}=[3,7621324,66]$, having the MPC a linear model that not correctly represents the dynamic of the system at the new operation point. The constraints and the EIS set remain unmodified respect to the previous section, although the was centered at the new operating point. The excitation procedure begins once the system is inside the ISI set, whit an excitation signal (PRBS) centered at the new operating point, with a variation of $20 \%$ around it. The trajectory of the system going from the original to the new operating point is guided by the MPC in the control operation mode (which is a classic MPC zone controller), and it is not shown for brevity. To preform the identification, the subspace identification technique [15] is used. The linear state space model obtained by this identification reaches a FIT of $80 \%$ about both outputs of the system. Two points should be

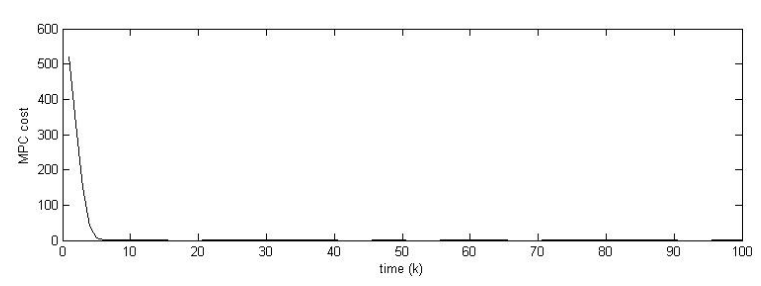

Figura 4: Cost evolution. 


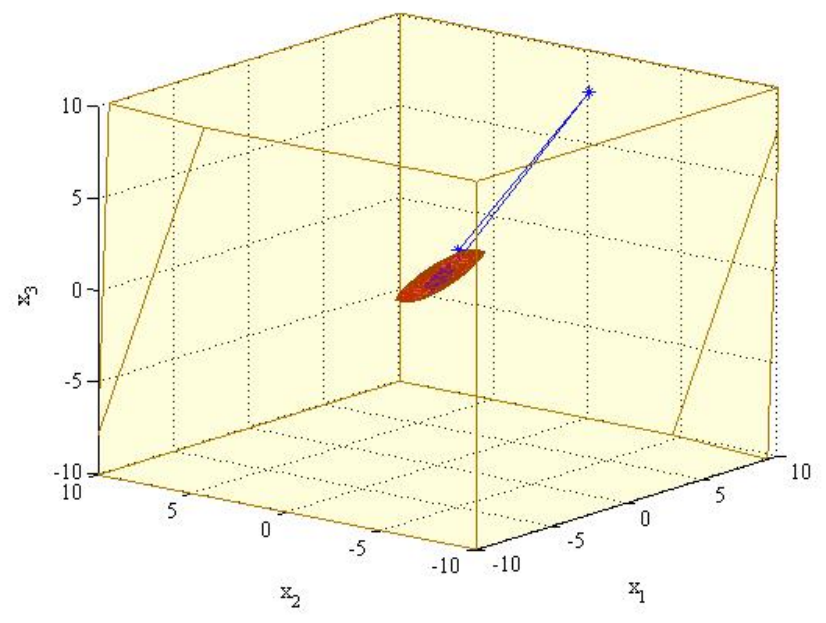

Figura 5: State evolution corresponding to a reidentification mode under a perturbation

remarked at this stage: first, in Figure 5 can be observed that while the excitation procedure is performed, the state trajectory do not leave the nominal ISI set (that was computed based on the original linear system). Second, if we inject a disturbance in $y_{s s}^{2}$, at time $\mathrm{k}=40$, that steers the state out of the ISI set, the MPC in the Re-identification operation mode aborts the identification procedure and guide the state back to ISI set. Then, once the system is again in the target ISI set, the identification procedure is resumed.

\section{Conclusions}

In this work, some results of an Application of Model predictive control suitable for closed loop reidentification to a polymerization reactor was presented. The proposed MPC guarantees persistent excitation when the system is inside a target region and guarantees recursive feasibility and closed-loop stability to this target region when the system is outside.

The key concept to mixture these two opposite objectives is the concept of attractivity of a robust invariant set, inside which the excitation of the system can be made.

From a practical point of view, a main advantage of the method is that the identification procedure can be made as in open-loop fashion, since once the system is inside the target region, no control actions affect the system. Future research clearly includes the study of the relation between the invariant target region and the exciting input set, in order to obtain a less conservative formulation.

\section{Referencias}

[1] A. H. González, A. Ferramosca, G. A. Bustos, J. L. Marchetti, and D. Odloak, "Model predictive control suitable for closed-loop re-identification," in Proceedings of the ACC, 2013.

[2] T. Soderstrom and P. Stoica, System identification, 1st ed. Prentice-Hall, 1989.
[3] H. Genceli and M. Nikolaou, "New approach to constrained predictive control with simultaneous model identification," AIChE Journal, vol. 42, pp. 2857-2868, 1996.

[4] S. L. Ballin, Controlador preditivo multivariavel com restricoes de excitacao para identificacao de processos em malha fechada. Universidade de Sao Paulo, 2008, tesis de Mestrado.

[5] O. Sotomayor, D. Odloak, and L. Moro, "Closedloop medel re-identification of processes under mpc whit zone control," Control Engineering Practice, vol. 17, pp. 551-563, 2009.

[6] G. Bustos, A. González, and J. Marchetti, "Monitoring steady-state gains under closed loop operation," Reunión de Trabajo Procesamiento de la Informacón y Control, vol. 14, pp. 875-880, 2011.

[7] L. Ljung, System identification-theory for the user, 2nd ed. Prentice-Hall, 1999.

[8] I. Kolmanovsky and E. G. Gilbert, "Theory and conmputation of disturbance invariant sets for discretetime linear systems," Mathematical Problems in Engineering: Theory, Methods and Applications, vol. 4, pp. 317-367, 1998.

[9] A. Ferramosca, D. Limon, A. H. González, D. Odloak, and E. F. Camacho, "MPC for tracking zone regions," Journal of Process Control, vol. 20, pp. 506-516, 2010.

[10] A. H. Gonzalez and D. Odloak, "A stable MPC with zone control," Journal of Process Control, vol. 19, pp. 110-122, 2009.

[11] L. Alvarez and D. Odloak, "Optimization and control of a continuous polymerization reactor," Brazilian Journal of Chemical Engineering, vol. 29, pp. 807-820, 2012.

[12] R. Jaisinghani and W. Ray, "On the dynamic behaviour of a class of homogeneous continuous stirred tank polymerization reactor," Chem. Eng. Sci., vol. 32, pp. 811-825, 1977.

[13] P. Hidalgo and C. Brosilow, "Nonlinear model predictive control of styrene polymerization at unstable operating points." Comp. Chem. Eng., vol. 14, pp. 481-494, 1990.

[14] B. Maner, F. D. III, B. Ogunnaike, and R. Pearson, "Nonlinear model predictive control of a simulated multivariable polymerization reactor using second-order volterra models," Automatica, vol. 32, pp. 1285 - 1301, 1996.

[15] P. V. Overschee and B. D. Moor, Subspace Identification for linear systems: theory, implementation, applications. Kluwer Academic Publishers, 1996. 